\title{
CORRIGENDUM
}

\section{Common disorders are quantitative traits}

Robert Plomin, Claire M. A. Haworth and Oliver S. P. Davis

Nature Rev. Genet. 10, 872-878 (2009)

An incorrect version of this article was previously published online (publication date 27 October 2009). In the second paragraph of the 'Identifying quantitative mechanisms' section on page 874 in this article, the history of genome-wide association (GWA) studies for type 2 diabetes was incorrectly described and a key reference was omitted. The corrected paragraph is shown below.

The authors apologize for this error.

For some traits, such as type 2 diabetes (T2D), a quantitative approach has already been embraced, with striking results ${ }^{9}$. Although the first T2D GWA studies were case-control studies (REF. 49, and subsequently other studies, for example, REF. 3), a wave of follow-up studies have focused on quantitative traits that are related to T2D, including levels of fasting glucose ${ }^{10}$ and C-reactive protein ${ }^{11}$, and glucose tolerance ${ }^{9}$. These studies are leading to refinements in the definition of T2D.

Reference 49 has now been added to the reference list. 